\title{
New Traveling Type 3D Full Body Scanner
}

\author{
Kohei IKE*a, Masaki HAYASHI ${ }^{a, b}$, Hideto KAMESHIMA ${ }^{a}$, Yuji NISHIO ${ }^{a}$, Yukio SATO ${ }^{a, b}$ \\ ${ }^{a}$ Spacevision Inc., Tokyo, Japan; ${ }^{b}$ Keio University, Tokyo, Japan
}

http://dx.doi.org/10.15221/13.182

\begin{abstract}
In this paper, a newly developed 3D full body scanner is descried. The new traveling type scanner is the most compact 3D full body scanner ever of all time, and it consists of three towers (Fig. 1(a)). By developing a much smaller new 3D camera (projector and video camera), each tower became significantly lighter and thinner compared to that of our previous body scanner. Moreover, each tower can be disassembled into three separated modules each having one 3D camera (Fig. 1(b)). In addition, by providing a CPU in each tower, 3D data obtained at each tower is decentrally processed allowing the use of a laptop PC as a control computer, which realizes real portability of the scanner system. Moreover, an interpolation program of scanning data was developed so that the scanner can output 3D human body data without deficits.
\end{abstract}

Keywords: 3D full body scanning, portable, accurate, high-speed interpolating.

System: The new scanner consists of three towers. It can capture both 3D human body shape and color texture of the human subject just in 2 seconds. Each tower consists of three vertically-stacked projector-camera modules and a base (Fig. 1(c)). Since our unique calibration technology [2] assures the alignment of the 3D shapes taken from each module, a precise placement of the towers is not required at the installation. Therefore, anyone can set up the scanner system in about 30 minutes alone. In this sense, the new body scanner is literally PORTABLE.

In addition, our interpolation program is applied to the obtained 3D data automatically. Since the program carries out fitting of a 3D human body model template to the obtained data, a unified 3D body scan data without deficits (Fig. 1(d)) can be created.

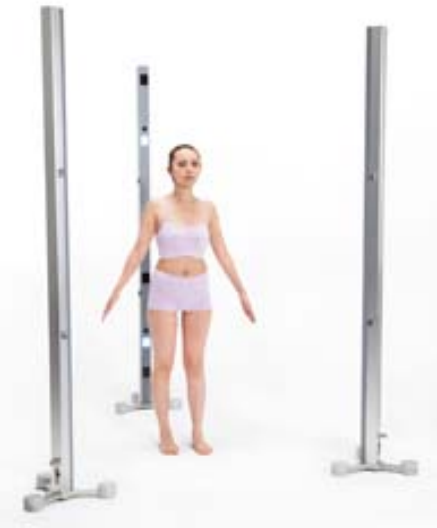

(a)

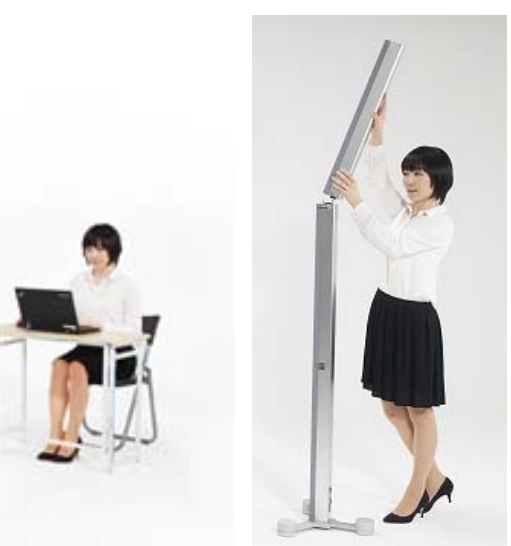

(b)

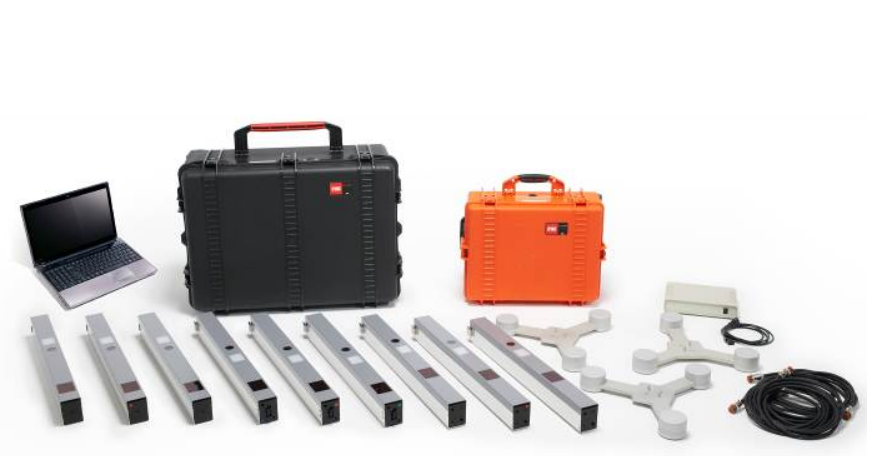

(c)

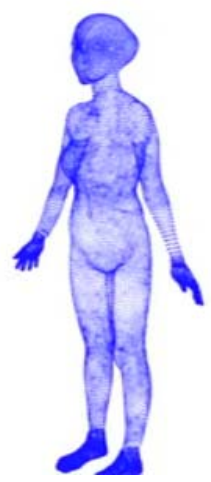

(d)

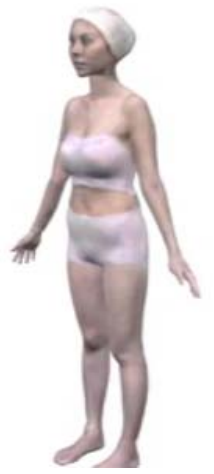

Fig. 1: New traveling type 3D full body scanner. (a) During scanning.

(b) Each tower can be disassembled into three modules. (c) All components.

(d) The example of interpolated data. (Point cloud / Closed surface with texture)

\footnotetext{
* ike@space-vision.jp; +81- 3- 5428-4144; www.space-vision.jp
} 
Advantages: Compared to our previous body scanner [3], the new traveling type 3D full body scanner has the following advantages:

\section{(1) Separable into smaller modules.}

Each tower of the new scanner can be disassembled into three compact projector-camera modules. This improves the portability of the new scanner. Reassemble of the tower is also so easy that one can reassemble these modules into a tower again by just snapping the module back (Fig. 1(b)).

\section{(2) Lighter and thinner modules.}

To realize real transportability of 3D body scanner, we developed a new projector that is much smaller compared to that of the current body scanner. Thanks to the new projector, each module now weighs only about $2.0 \mathrm{~kg}$ and its size is only $6 \mathrm{~cm}$ (width) $\times 8 \mathrm{~cm}$ (depth) $\times 68 \mathrm{~cm}$ (height) (Fig. 1(c)). Owing to the separable tower and each light and thin module, our new scanner has become the most compact 3D full body scanner of the world. The portability of our new scanner is fulfilled by our unique calibration technique which ensures quick and accurate alignment of the towers after installation at a new site.

(3) Distributed system controlled from a laptop PC.

For our current body scanner, IEEE 1394 cables were used for connecting video cameras, and therefore, slots for multiple IEEE boards are required for system control PC. To make the system more compact by changing the desktop PC to a laptop PC for system control, and have a simpler network cable connection, we changed the scanner system to a distributed system. A CPU is installed in each tower to control the three projector-cameras within the tower, and a standard laptop PC can be used as system control PC. The control laptop PC communicates with those CPUs in each tower via Ethernet cables. By switching to the distributed system, the new scanner can be operated by a simple laptop PC and more cable-free installation is achieved (Fig. 1(a)).

(4) Interpolation of the scanned body data.

The new body scanner is equipped with software that interpolates scanned body data. We developed the software based on an algorithm called "mHBM (markerless Homologous Body Modeling)" [5]. This software can carry out fitting of a 3D human body model template to the scanned data automatically. Thereby, you can get a unified 3D body scan data in which deficit portions were smoothly interpolated, while maintaining the form of scanned data (Fig. 1(d)).

\section{References}

1. Koichiro Yamauchi, Yukio Sato, "3D Human Body Measurement by Multiple Range Images”, 18th International Conference on Pattern Recognition, Hong Kong, vol.4, pp.833-836, Aug. 2006.

2. Hideto Kameshima and Yukio Sato, "A Calibration Method for Multiple Rangefinders System", IS\&T/SPIE 19th Annual Symposium Electronic Imaging (EI2008), Proceedings of SPIE, vol.6805, pp. 68050P-68050P-8, San Jose, Jan. 2008.

3. Hideto KAMESHIMA, Yuji NISHIO, Yukio SATO, "A Portable 3D Body Scanner", International Conference on 3D Body Scanning Technologies, Lugano, Switzerland, pp.191-196, October. 2010.

4. M. Hayashi, H. Kameshima, Y. Nishio, Y. Sato, "A portable and compact 3D body - 3D body scanner traveling type -", $2^{\text {nd }}$ International Conference on 3D Body Scanning Technologies, Lugano, Switzerland, pp.284-285, October. 2011.

5. S. Yamazaki, M. Kouchi, M. Mochimaru, "Markerless landmark localization on body shape scans by non-rigid model fitting", $2^{\text {nd }}$ International Digital Human Modeling Symposium, Michigan, \#43, June. 2013. 